\title{
Validity of a 5-minute focused echocardiography with A-F mnemonic performed by non-echocardiographers in the management of patients with acute chest pain
}

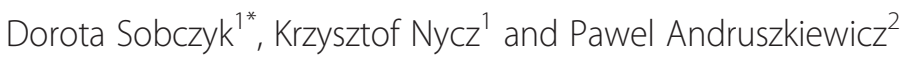

\begin{abstract}
Study objective: To validate the practicality of focused echocardiography with A-F mnemonic performed by non-specialists in patients with suspected acute coronary syndrome (ACS).

Design: This prospective observational study was conducted in the Emergency Room within 12 months period. Study population consisted of consecutive patients with preliminary diagnosis of an ACS.

The following data were analyzed: demographics, clinical condition, medical history, ECG, transthoracic echocardiography (TTE) and levels of cardiac necrotic markers. TTE was performed within the first 15 minutes after the admission by the resident on-call. TTE images were interpreted and reported with mnemonic A-F. All studies were recorded and reviewed within 24 hours by the cardiologist.
\end{abstract}

Results: 1312 consecutive patients were enrolled to the study. TTE with A-F mnemonic revealed: RWMAs in $82,87 \%$ patients with confirmed ACS, other significant cardiac pathologies were found in $2,21 \%$ in ACS and $46,52 \%$ in non-ACS groups respectively. On the basis of these findings, 20 (1,92\%) ACS and 29 (10,62\%) non-ACS group patients underwent target operative treatment. Survey showed that both echocardiographic image acquisition and its interpretation with A-F mnemonic, took less than 5 minutes in $95 \%$ of cases. Residents found A-F mnemonic algorithm simple and useful. No differences were found in key findings between TTE performed by resident and the cardiologist.

Conclusion: Focused echocardiography with A-F mnemonic allows both confirmation of acute myocardial ischemia and detection of the other life-threatening cardiac conditions resulting in proper bedside decision of directed treatment. Mnemonic based TTE enables reliable examination by properly trained residents.

Keywords: Acute coronary syndrome, Emergency room, Focused echocardiography, Emergency echocardiography, A-F mnemonic

\section{Introduction}

The workup of chest pain management (including suspected ACS) includes: clinical presentation, medical history, physical examination, risk stratification, laboratory tests, and non-invasive imaging [1-4]. Among non-invasive imaging techniques transthoracic echocardiography (TTE) plays a pivotal role in acute setting [5-7]. However, full

\footnotetext{
*Correspondence: dorotasobczyk@yahoo.com

${ }^{1}$ The Department of Interventional Cardiology, John Paul 2nd Hospital, Pradnicka 80, 31202 Cracow, Poland

Full list of author information is available at the end of the article
}

echocardiographic examination is time-consuming. It is usually performed by a cardiologist with an expertise in clinical echocardiography who is not easily available at the time of emergency admission. In such situation when time is an important issue, introduction of a focused, simplified echocardiographic scheme for primary screening and reporting is desirable. Such algorithm should be easy to perform, reproducible, directed at the most common lifethreatening pathologies and possible to learn by a nonspecialist during a short training. The A-F mnemonic 
meets all of these criteria and could be easy implemented in the emergency room settings [8].

\section{Study objective}

The purpose of the present study was to validate the usefulness and practicality of focused transthoracic echocardiography with A-F mnemonic, performed by nonechocardiographers in patients with acute chest pain.

\section{Material and methods}

The observational study was approved by the Local Ethical Committee of John Paul 2nd Hospital in Cracow (ref. number 792) and informed consent was obtained from all study participants.

The study was conducted in the Emergency Room of the Interventional Cardiology Department of John Paul 2nd Hospital in Cracow, within 12 months period (January 2013 to January 2014).

We prospectively enrolled all patients admitted to the Emergency Room with preliminary diagnosis of an acute coronary syndrome made by the pre-hospital health care providers. The diagnosis was based on typical clinical symptoms and ECG findings: new or presumed new significant ST-segment-T-wave (ST-T) changes, new left bundle branch block (LBBB) or development of pathological Q waves. Exclusion criteria were: age $<18$ years and difficult acoustic window resulting in inability to obtain interpretable ultrasound images. 59 patients (59/1371, 4.3\%) were excluded from final analysis because of difficult acoustic window and un-interpretable ultrasound images.

14 residents (at least after the completion of third year of training) were included in the study. All of them had basic skills in TTE and performed at least 75 examinations before participation in the study. Additionally, they underwent a minimum of 30-minutes didactic and 1,5hour structured hands-on dedicated practice with the AF examination scheme.

In the Emergency Room, clinical condition was assessed according to both Canadian Cardiovascular Society (CCS) and New York Heart Association (NYHA) classifications. Prior myocardial infarction, coronary revascularization and pre-hospital cardiac arrest, were noted. In order to confirm the diagnosis of ACS, further investigations were instituted including: ECG, transthoracic echocardiography (TTE) and blood tests for measurement of biomarkers for myocardial damage.

Electrocardiogram (ECG) was performed within $5 \mathrm{mi}-$ nutes after the admission to the Emergency Room and repeated in 15-minutes intervals in symptomatic patients with an initial non-diagnostic ECG and during every recurrence of symptoms. The ECG recording included: standard 12-leads, right precordial leads (V3R, V4R) and posterior leads (V7-V9) when appropriate.
According to newly introduced institutional fast-track protocol, transthoracic bedside echocardiography was performed within the first 15 minutes after patient's presentation, by the resident on-call. Duration of the examination was recorded and usefulness of the mnemonic was assessed by the physicians. TTE images were interpreted with the simplified mnemonic A-F, introduced in our Emergency Room in order to standardize bedside cardiac examination. In A-F mnemonic, consecutive letters of an alphabet represent a particular anatomical structure or measure of cardiac function: A-aorta, B-both ventricles, C-contractility, D-dimensions, E-effusion, F-further abnormalities (Table 1). The examinations were conducted with portable ultrasound system equipped with a 1-5 MHz transthoracic phased-array transducer (Vivid I, GE Healthcare, USA, and CX 50, Philips, Eindhoven, Netherlands). Heart was visualized in 5 basic echocardiographic views: parasternal long axis, parasternal short axis, apical four-chamber, apical two-chamber and subcostal. All studies were recorded as digital 10-seconds video clips and reviewed within 24 hours by the consultant cardiologist unaware of the resident's conclusions. For the purpose of the study, the cardiologist was asked to summarize his/her findings using AF-mnemonic tool structure and if necessary to add important information extending beyond this scheme. Both summaries were confronted by independent researcher (experienced cardiologist-echocardiographer) during the analysis and the decision was made if any discrepancies between the two diagnoses might have had any impact for the choice of treatment and the final outcome.

Blood samples were collected for plasma levels of biomarkers of myocardial damage: creatine kinase (CK), cardiac isoenzyme of creatine kinase (CK-MB) and high sensitivity troponin $\mathrm{T}$ (hsTnT), at the time of admission and, if necessary, by repeated measurements every 3-6 hours within the first day of hospitalization. Blood tests were assayed by routine automated laboratory techniques (Cobas System 6000, Roche Diagnostics GmbH, Manheim, Germany). All biochemical analyses were performed in the central hospital laboratory, certified with cardiac and clinical chemistry program by RIQAS (Randox International Quality Assessment Scheme, UK).

Coronary angiogram was performed in the following groups of patients: with typical chest pain and typical ischemic ECG changes (as stated above), regardless of cardiac biomarkers on admission; with atypical clinical presentation (atypical chest pain, non-diagnostic ECG) but with elevated cardiac biomarkers on admission and/or typical evolution (rise or fall) of biomarkers in following 3-6 hours; with atypical clinical presentation (atypical chest pain, non-diagnostic ECG) and negative cardiac biomarkers, but with multiple risk factors (e.g. hypertension, dyslipidemia, diabetes, previous MI, previous revascularization, positive family history) and new or presumed new 
Table 1 Description of the mnemonic A-F algorithm

\begin{tabular}{|c|c|c|c|}
\hline Letter & Description & Question & Possible diagnosis (if the answer is YES) \\
\hline \multirow[t]{3}{*}{$A$} & Aorta & Is aortic root dilated? & Aortic root dilatation/aneurysm \\
\hline & & Is proximal aortic diameter $>4 \mathrm{~cm}$ ? & Ascending aortic dilatation/aneurysm \\
\hline & & Is dissection flap seen? & Aortic dissection \\
\hline \multirow[t]{3}{*}{ B } & Both ventricles & Is there RV overload present? & Pulmonary embolism \\
\hline & & $\mathrm{RV} / \mathrm{LV}>1$ & Pulmonary hypertension \\
\hline & & D-sign & RV infarction \\
\hline \multirow[t]{5}{*}{ C } & Contractility & Is LV contractility impaired? & \\
\hline & & Depressed global systolic function & LV heart failure \\
\hline & & Regional wall motion abnormalities & Acute myocardial infarction \\
\hline & & Is RV contractility impaired? & \\
\hline & & Depressed global systolic function & RV heart failure; RV infarction; pulmonary embolism \\
\hline \multirow[t]{6}{*}{ D } & Dimensions & Are there any abnormal dimensions? & \\
\hline & & Ascending aorta $>4 \mathrm{~cm}$ & Aortic dilatation/aneurysm (look for aortic dissection) \\
\hline & & LV end-diastolic dimension $>6 \mathrm{~cm}$ & LV dilatation (assess global LV function) \\
\hline & & RV end-diastolic dimension $>4.2 \mathrm{~cm}$ & RV dilatation (look for the RV overload) \\
\hline & & $\mathrm{LA}$ anteroposterior dimension $>4.5 \mathrm{~cm}$ & LA dilatation \\
\hline & & RA major $>5.4 \mathrm{~cm}$ and/or minor dimension $>4.4 \mathrm{~cm}$ & RA dilatation (look for RV overload) \\
\hline \multirow[t]{6}{*}{ E } & Effusion & Is pericardial effusion present? & Pericardial effusion \\
\hline & & Are there any signs of cardiac tamponade? & Cardiac tamponade \\
\hline & & RA end-systolic or diastolic collapse & \\
\hline & & RV diastolic collapse & \\
\hline & & Vena cava plethora & \\
\hline & & Is there pleural effusion? & Pleural effusion \\
\hline $\mathrm{F}$ & Further abnormalities & Any other abnormal findings not listed above? & \\
\hline
\end{tabular}

From: Sobczyk and Andruszkiewicz P [8].

regional wall motion abnormalities in TTE on admission (performed according to A-F mnemonic) (Figure 1).

Medical data were analyzed independently by the authors and in each case a consensus was achieved. No ambiguous echocardiographic examinations were recorded, all results were clearly stated in a dichotomic manner: echocardiographic abnormality present or not.

Statistical analysis was performed using STATISTICA v 8.0 software. Numerical data were expressed as mean values \pm SD.

\section{Results}

1312 consecutive adult patients (858 male, age 21-98 years, mean age $67 \pm 12,32$ years) were enrolled to the study. Baseline demographic and clinical data are shown in Table 2. Echocardiographic findings are summarized in Table 3.

Chest pain was present in $98,1 \%$ of patients, with $51,68 \%$ of patients in 4 CCS class. Dyspnea was observed in $36,28 \%$ of patients, with $8,69 \%$ of patients in NYHA 4 class. ECG on admission revealed ST-depression in $56,55 \%$ and ST-elevation in $37,35 \%$ of patients (Table 2).
Troponin $\mathrm{T}$ levels were higher than 99th percentile of upper range limit (URL) in $87,42 \%$ of patients, exceeding three times URL in $64,94 \%$ of cases (Table 2). Coronary angiography was performed in $95,05 \%$ of patients. $75,3 \%$ of them had subsequent coronary revascularization (PCI or $\mathrm{CABG}$ ). The final diagnosis of myocardial infarction (with or without persistent ST-segment elevation), unstable angina (UA) or Takotsubo cardiomyopathy, was determined according to the recent clinical guidelines (the group with confirmed ACS). Final ACS diagnosis was confirmed in $79,19 \%$ of enrolled patients.

Echocardiography performed on admission showed regional wall motion abnormalities (RWMAs), with normal wall thickness and no features of post-infarction scar, in 99,5\% of patients with STEMI and 78,5\% with NSTEMI, and it was considered an echocardiographic finding enhancing the probability of ACS. Coexisting cardiac pathology (apart from ACS) was revealed in 23 patients $(2,21 \%)$ with confirmed ACS. In 20 of them (86,96\%), with significant structural disease (at least moderate aortic stenosis, severe mitral regurgitation, post-infarction VSD), TTE was a decisive diagnostic tool determining the choice 


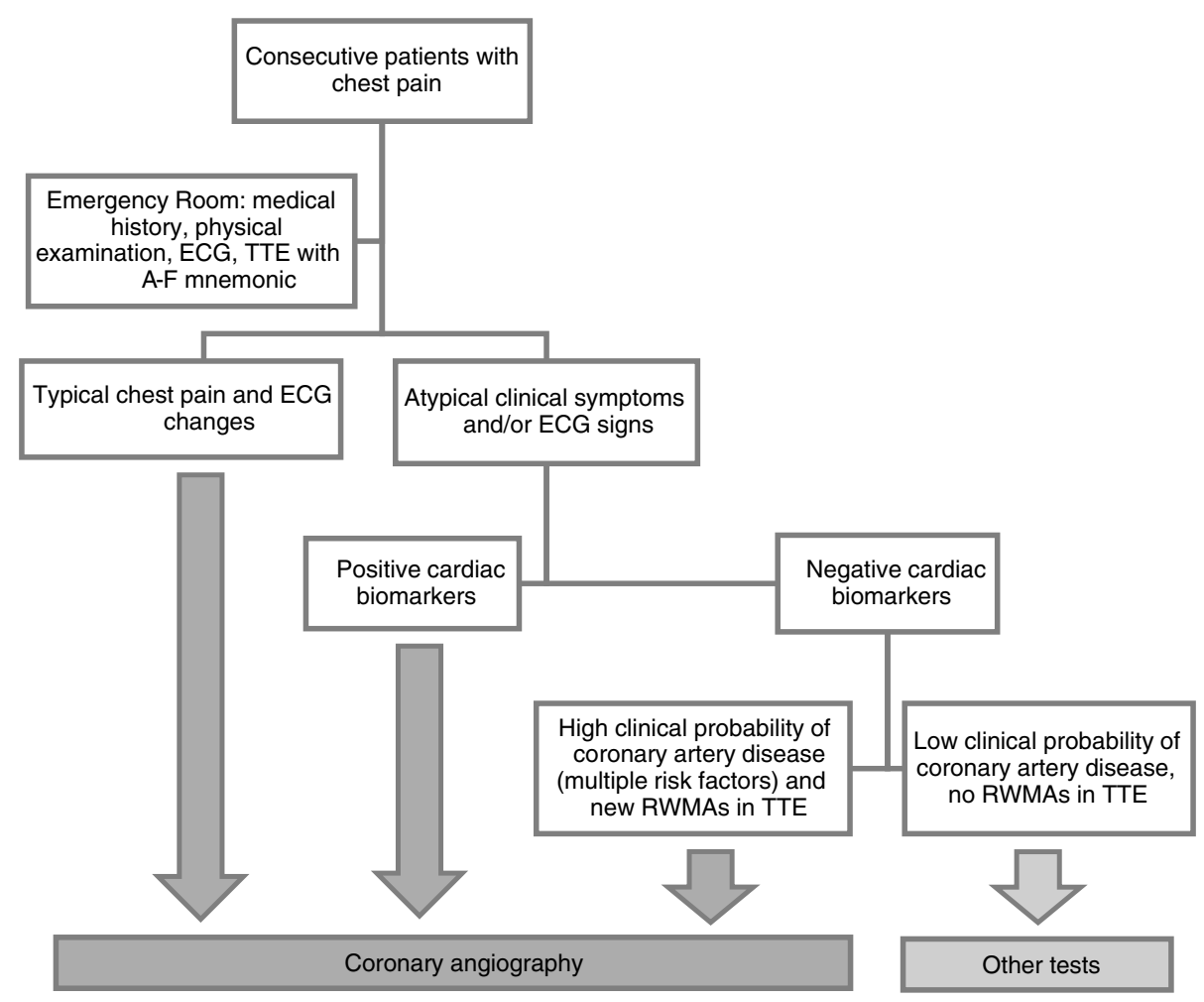

Figure 1 Flow chart of patients in the study.

of a treatment. After coronary angiography, all these patients underwent urgent coronary artery bypass grafting with additional target procedure: aortic valve replacement, mitral valve annuloplasty or VSD closure.

In patients in whom the initial suspicion of ACS was not finally confirmed, the final diagnosis was established based on echocardiography alone or additional diagnostic tests (blood tests, chest X-ray, computed tomography). In 127 patients (46,52\%) from this group, clinically significant echocardiographic abnormalities were found during the AF-mnemonic - based TTE exam (Table 4). On the basis of these findings, 29 patients $(10,62 \%)$ underwent invasive treatment: aortic valve replacement, mitral annuloplasty, aortic alloplasty, decompression of tamponade, VSD closure, fibrinolysis, removal of infected pacemaker electrode.

Survey of the residents involved in the study, showed that focused echocardiographic examination and its interpretation based on A-F mnemonic, took less than 5 minutes in $95 \%$ of cases. All of them found A-F mnemonic algorithm very useful in sticking to the examination structure and avoiding omissions of the important findings. No differences were found in key findings and conclusions between TTE performed by the resident on-call and the cardiologist (Table 5). In 242/1312 (18,45\%) cases cardiologists reported extra findings extending beyond the A-F scheme (mild mitral valve regurgitation, mild or moderate tricuspid valve regurgitation, mild left atrial enlargement). According to the cardiologist in charge of the study none of these, however, had any impact on the choice of the instituted treatment.

\section{Discussion}

According to recent clinical guidelines echocardiography plays an important role as a first-line emergency diagnostic tool in patients with chest pain (including suspected ACS) [2-5]. It is a quick and safe method to establish dynamic cardiac anatomy and pathophysiology. Echocardiographic evidence of new regional wall motion abnormalities is one of the diagnostic criteria for an acute myocardial infarction (when combined with detection of rise and/or fall of cardiac biomarkers) [2] whereas impaired left ventricular global systolic function is a factor related with a poor prognosis. Moreover, TTE can facilitate differential diagnosis of various diseases related with chest pain [2-4,6,7,9-13]. Several life-threatening conditions (acute aortic dissection, acute pulmonary embolism, cardiac tamponade, severe valvular disease or hypertrophic cardiomyopathy) requiring urgent treatment, can be identified with this bedside tool [9-13].

Although some might argue that TTE has no added value in the non-shocked patient, all recent clinical guidelines for diagnosis and management of myocardial infarction, emphasize the role of TTE as the initial imaging modality in patients with acute chest pain 
Table 2 Demographic and clinical characteristics of the study population

\begin{tabular}{ll}
\hline Characteristic & Value \\
\hline Age (years) & $67 \pm 12,32$ \\
Male sex, n (\%) & $858(65,39)$ \\
CCS class & $3,38 \pm 0,73$ \\
NYHA class & $1,64 \pm 0,97$ \\
Concomitant diagnosis, n (\%): & \\
Prior myocardial infarction & $268(20,43)$ \\
Prior PCI & $257(19,59)$ \\
Prior CABG & $70(5,34)$ \\
Prior AVR & $12(0,91)$ \\
Pre-hospital cardiac arrest, n (\%) & $76(5,79)$ \\
ECG changes at admission, n (\%): & \\
ST depression & $742(56,55)$ \\
ST elevation & $490(37,35)$ \\
Q/QS complex & $213(16,23)$ \\
Negative T-waves & $161(12,27)$ \\
LBBB & $49(3,73)$ \\
Other & $167(12,73)$ \\
hs TnT (ng/ml) & $0,41 \pm 0,86$ \\
CPK (U/l) & $452,96 \pm 2256,02$ \\
CM-MB (U/l) & $40,86 \pm 54,23$ \\
\hline PCl-percutan & \\
\hline
\end{tabular}

$\mathrm{PCl}$-percutaneous coronary intervention; CABG-coronary artery bypass grafting AVR-aortic valve replacement; LBBB-left bundle branch block; hs TnT-high sensitivity troponin T; CPK-creatine kinase; CK-MB-cardiac isoenzyme of creatine kinase.

[2-4,12-14]. Everyday emergency practice in our center also confirms the importance of this diagnostic procedure. We have observed numerous patients with typical chest pain and ST-segment changes (also ST elevation) caused by other than ACS, life-threatening conditions that could have been detected by focused TTE, i.e. acute aortic dissection, cardiac tamponade or acute pulmonary embolism. In this subset of patients, immediate coronary angiography may expose them to an unnecessary risk (i.e. catheterization of false lumen in patients with acute proximal aortic dissection) and finally delay appropriate treatment. That is why we believe that echocardiography should be routinely available in Emergency/Admission Room and implemented in all patients with suspected ACS (if it is possible without delay of target treatment). Based on both current recommendations and own experience, focused TTE with A-F mnemonic was introduced in our hospital as an integral and mandatory part of an initial evaluation of all patients admitted due to chest pain/ suspected ACS. It must be highlighted that bedside TTE with A-F mnemonic is not equivalent and does not replace the comprehensive echocardiographic examination, performed as recommended by the guidelines.
Table 3 Echocardiographic findings in study population

\begin{tabular}{|c|c|c|}
\hline $\begin{array}{l}\text { Echocardiographic } \\
\text { mnemonic }\end{array}$ & Findings & Value \\
\hline \multirow[t]{2}{*}{ A (aorta) } & Aortic dilatation, n (\%) & $62(4,73)$ \\
\hline & Aortic dissection, n (\%) & $5(0,38)$ \\
\hline \multirow[t]{2}{*}{ B (both ventricles) } & Right ventricular dilatation, n (\%) & $46(3,51)$ \\
\hline & Right ventricular overload, n (\%) & $20(1,52)$ \\
\hline \multirow[t]{3}{*}{ C (contractility) } & $\begin{array}{l}\text { Regional wall motion disturbances, } \\
\mathrm{n}(\%)\end{array}$ & $921(70,19)$ \\
\hline & LVEF (\%) & $46,569 \pm 13,41$ \\
\hline & LVEF $\leq 30 \%, n(\%)$ & $199(15,17)$ \\
\hline \multirow[t]{5}{*}{ D (dimensions) } & Abnormal heart dimensions, n (\%) & $325(24,77)$ \\
\hline & Left ventricular dilatation, n (\%) & $71(5,41)$ \\
\hline & Left ventricular hypertrophy, n (\%) & $145(11,05)$ \\
\hline & Left and right atrial dilatation, n (\%) & $104(7,97)$ \\
\hline & Right ventricular dilatation, n (\%) & $54(4,12)$ \\
\hline \multirow[t]{4}{*}{ E (effusion) } & Pericardial or pleural effusion, n (\%) & $61(4,65)$ \\
\hline & Pericardial effusion, n (\%) & $48(3,66)$ \\
\hline & Cardiac tamponade, n (\%) & $3(0,23 \%)$ \\
\hline & Pleural effusion, n (\%) & $18(1,37)$ \\
\hline \multirow{4}{*}{$\begin{array}{l}\text { F (further } \\
\text { abnormalities) }\end{array}$} & Further abnormalities, n (\%) & $242(18,45)$ \\
\hline & Mitral valve abnormalities, n (\%) & $126(9,60)$ \\
\hline & Aortic valve abnormalities, n (\%) & $77(5,87)$ \\
\hline & Other, n (\%) & $78(5,95)$ \\
\hline$A+B+E+F$ & Any abnormality & $319(24,31)$ \\
\hline Normal study & No abnormalities & $74(5,64)$ \\
\hline
\end{tabular}

LVEF-left ventricular ejection fraction.

Performing TTE should not delay direct treatment of suspected ACS. In all patients with typical clinical manifestation and ST elevation on ECG, emergency coronary angiography and subsequent revascularization should be initiated as soon as possible. However, limited bedside TTE usually does not take more than five minutes and may be performed simultaneously with other procedures on admission (ECG recording, collecting blood samples or preparation for coronary angiography). High prevalence of other cardiac and non-cardiac life-threatening conditions mimicking the clinical presentation of ACS, justifies the implementation of additional imaging tests in the management of patients with suspected ACS. In our study population, final ACS diagnosis was confirmed in $79,19 \%$ of enrolled patients.

Correct and quick diagnosis of a suspected acute coronary syndrome at admission determines outcome of the treatment. However, unquestionable value of TTE may be limited by the lack of skill and competency in the physicians. The full echocardiographic examination seems to be too complicated, extensive and time-consuming and some important pathologies may be overlooked and 
Table 4 Final diagnosis of patients admitted with the primary diagnosis of ACS with the prevalence of typical echocardiographic findings reported by limited TTE with A-F mnemonic

\begin{tabular}{|c|c|c|c|}
\hline Final diagnosis & $\begin{array}{l}\text { Number of } \\
\text { patients (\%) }\end{array}$ & Echocardiographic findings & $\begin{array}{l}\text { Echocardiographic } \\
\text { diagnosis, } \mathrm{n}(\%)\end{array}$ \\
\hline Confirmed ACS & $1039(79,19)$ & RWMA & $861(82,87)$ \\
\hline NSTEMI & $543(52,26)$ & RWMA & $426(78,45)$ \\
\hline STEMI & $387(37,25)$ & RWMA & $385(99,48)$ \\
\hline UA & $92(8,85)$ & RWMA & $33(35,87)$ \\
\hline Takotsubo cardiomyopathy & $17(1,65)$ & RWMA, apical ballooning & $17(100)$ \\
\hline Adjective diagnosis in confirmed ACS & $23(2,21)$ & listed below & $23(100)$ \\
\hline Moderate/severe aortic stenosis & $16(1,54)$ & AV calcification & $16(100)$ \\
\hline Severe mitral regurgitation & $3(0,29)$ & MR jet & $3(100)$ \\
\hline Decompensated CHF & $3(0,29)$ & $\begin{array}{l}\text { dilated LV, LA, small pericardial/pleural } \\
\text { effusion, low LVEF }\end{array}$ & $3(100)$ \\
\hline Ventricular septal defect & $1(0,09)$ & IVS interruption & $1(100)$ \\
\hline Non-confirmed ACS & $273(20,81)$ & any abnormality & $151(55,31)$ \\
\hline Decompensated CHF & $51(18,68)$ & $\begin{array}{l}\text { dilated LV, LA, small pericardial/pleural } \\
\text { effusion, low LVEF }\end{array}$ & $51(100)$ \\
\hline Stable CAD & $35(12,92)$ & RWMA & $0(0)$ \\
\hline Acute pulmonary embolism & $21(7,69)$ & RV overload & $20(95,27)$ \\
\hline Aortic valve disease & $15(5,49)$ & AV calcification & $15(100)$ \\
\hline Myocarditis & $10(3,66)$ & moderately impaired global LV contractility & $8(80)$ \\
\hline Tachycardia (AF, SVT) & $10(3,66)$ & atrial dilatation & $8(80)$ \\
\hline Pericarditis & $8(2,93)$ & pericardial effusion, thickened pericardium & $8(100)$ \\
\hline Prinzmetal angina & $8(2,93)$ & none & $8(100)$ \\
\hline Pneumonia & $6(2,19)$ & small pleural effusion & $4(66,666)$ \\
\hline Mitral valve disease & $5(1,83)$ & MR jet & $5(100)$ \\
\hline Aortic dissection & $5(1,83)$ & dilated aorta, dissection flap & $5(100)$ \\
\hline Hypertensive crisis & $6(2,19)$ & $\mathrm{LVH}$ & $6(100)$ \\
\hline Exacerbated COPD & $4(1,47)$ & RV dilatation & $4(100)$ \\
\hline $\mathrm{HOCM}$ & $4(1,47)$ & severe LVH, LVOTO & $4(100)$ \\
\hline Cardiac tamponade & $3(1,09)$ & pericardial effusion, RA and RV collapse & $3(100)$ \\
\hline Ventricular septal defect & $2(0,73)$ & IVS interruption & $2(100)$ \\
\hline Hypovelemic shock & $1(0,37)$ & small LV, IVC $<1 \mathrm{~cm}$ & $1(100)$ \\
\hline Infective endocarditis associated with pacemaker electrode & $1(0,37)$ & abnormal structures on the pacemaker electrode & $1(100)$ \\
\hline Perivalvular leak in AVR & $1(0,37)$ & perivalvular jet & $1(100)$ \\
\hline Lung tumor & $1(0,37)$ & none & $1(100)$ \\
\hline Acute pancreatitis & $1(0,37)$ & none & $1(100)$ \\
\hline Exacerbated polimyositis & $1(0,37)$ & none & $1(100)$ \\
\hline Other & $71(26,01)$ & no abnormal findings & $71(100)$ \\
\hline
\end{tabular}

ACS-acute coronary syndrome; NSTEMI-non-ST-elevation myocardial infarction; STEMI-ST-elevation myocardial infarction; UA-unstable angina; RWMAs-regional wall motion abnormalities; AV-aortic valve; MR-mitral regurgitation; LA-left atrium; LV-left ventricle; IVS-inteventricular septum; RV-right ventricle; RA-right atrium; IVC-inferior vena cava; CHF-chronic heart failure; COPD-chronic obstructive pulmonary disease; AVR-aortic valve replacement; HOCM-hypertrophic obstructive cardiomyopathy; LVH-left ventricular hypertrophy; LVOTO-left ventricular outflow tract obstruction.

omitted especially when distracting abnormalities are present. Thus echocardiographic diagnosis of ACS in an Emergency Room should concentrate on: evaluation of presence of new RWMAs; global left ventricular systolic function assessment (as a predictor of outcome); and identification of other pathologies leading to chest pain or dyspnea.

In order to improve the standard of care of patients with suspected ACS in our Emergency Room, we decided to introduce a new fast-track protocol based on 


\begin{tabular}{|c|c|c|}
\hline $\begin{array}{l}\text { A-F } \\
\text { mnemonic }\end{array}$ & Findings & $\begin{array}{l}\text { Concordance between } \\
\text { the reports (\%) }\end{array}$ \\
\hline $\mathrm{A}$ & Aortic dilatation & 100 \\
\hline$A$ & Intimal flap & 100 \\
\hline B & RV dilatation & 100 \\
\hline B & RV overload (RV > LV) & 100 \\
\hline C & RWMAs & 100 \\
\hline C & Apical balooning & 100 \\
\hline C & $\begin{array}{l}\text { Severely depressed global } \\
\text { LV function }\end{array}$ & 100 \\
\hline $\mathrm{D}$ & $\begin{array}{l}\text { Abnormal heart dimensions } \\
\text { (mild left atrial dimension) }\end{array}$ & 57,32 \\
\hline E & Pericardial effusion & 100 \\
\hline E & Pleural effusion & 100 \\
\hline $\mathrm{F}$ & $\begin{array}{l}\text { Further abnormalities (mild mitral } \\
\text { regurgitation, mild/moderate } \\
\text { tricuspid regurgitation) }\end{array}$ & 70,76 \\
\hline
\end{tabular}

RV-right ventricular; RWMAs-regional wall motion abnormalities; LV-left ventricular.

TTE performed by the residents on-call. Our present study confirmed the utility of early simplified TTE in ACS suspected patients. RWMAs were present in $82,87 \%$ of patients with confirmed ACS (with almost 100\% prevalence in STEMI and Takotsubo cardiomyopathy). The study showed that new RWMAs on echocardiography strongly supported ACS diagnosis and was a deciding factor for a further coronary angiography in patients with atypical clinical symptoms and/or atypical ECG changes. TTE with A-F mnemonic revealed significant cardiac pathology in 127 (46,52\%) non-ACS and 23 (2,21\%) ACS patients. On the basis of these findings, 20 (1,92\%) of ACS and $29(10,62 \%)$ of non-ACS group patients underwent target invasive treatment (aortic valve replacement, mitral annuloplasty, aortic alloplasty, decompression of tamponade, VSD closure, fibrinolysis, removal of infected pacemaker electrode).

All echocardiographic examinations were repeated within 24 hours by a cardiology consultant with expertise in echocardiography, and all digital clips recorded and described based on A-F mnemonic by residents, were reviewed by investigators blinded to the final diagnosis. Comparison of both examinations confirmed $100 \%$ coincidence of A-F-based findings. In almost $20 \%$ of cases cardiologists reported extra findings extending beyond the A-F scheme, but none of these however had any impact on the choice of the treatment. This excellent coherence between TTE reports done by residents and cardiologistsechocardiographers is puzzling. It may result from their competency in echocardiography. All residents had basic skills in echocardiography with at least 75 exams performed till the end of 2012 (mean 86 examinations/ resident).

Vignon et al. [15] in a prospective descriptive clinical study assessed the efficacy of limited tailored echocardiographic training for non-cardiology residents without any ultrasound experience. The authors proved that a 12hour training program was sufficient for reaching competence in basic critical care echocardiography. Mjolstad et al. [16] examined the feasibility and reliability of pocketsize hand-held echocardiography (PHHE) by medical residents with limited experience in ultrasound. Based on high correlation with reference method, authors concluded that focused examinations performed by residents after a training period, bring a reliable information and have the potential to improve in-hospital diagnostic procedures. Fredericksen et al. [17] compared the diagnostic accuracy of point-of-care (POC) echocardiography performed by a novices versus experienced cardiologists with an expertise in echocardiography, with regard to the assessment of six cardiac conditions: pericardial effusion, left ventricular (LV) dilatation, right ventricular dilatation, LV hypertrophy, LV failure and aortic stenosis. This study showed that a novice examiner was able to detect common and significant heart pathology in six different categories with good accuracy. Muniz Pazeli et al. [18] investigated whether a nephrologist with limited ultrasound training could accurately assess the volume status by inferior vena cava diameter in hemodialysis patients. The inter-examiner agreement between nephrologist and cardiologist was excellent.

Defined competence level is required to perform trustworthy examination. According to European Association of Echocardiography (EAE) [19] 350 examinations must be performed to achieve a basic level of competence. All residents participated in our study apart from a standard TTE training underwent a 1,5-hour structured hands-on dedicated practice with the A-F scheme practical implementation. Survey showed that both echocardiographic image acquisition and interpretation based on A-F mnemonic, usually took less than 5 minutes. All residents found A-F mnemonic algorithm very simple and useful in sticking to the examination structure and helpful in avoiding omissions of important findings.

It is well documented that focused bedside echocardiography can bring important anatomical and hemodynamic information in an acute setting. It can affect the patient's management, direct further diagnosis and modify therapeutic decision. Panaoulas et al. [20] proved a significant impact of point-of-care (POC) echocardiography on final clinical diagnosis in cardiac patients. Final-year medical students and junior doctors without prior ultrasound experience participated in the study after a 2-hours bedside tutorial. The use of POC echocardiography significantly improved the diagnostic accuracy in comparison to 
physical examination, medical history and ECG findings. Spencer et al. [21] also noted that POC echocardiography substantially improved the detection of important cardiovascular pathologies compared with physical examination. Manasia et al. [22] reported that limited TTE performed by intensivists, provided new information and altered further management in a significant number of intensive care patients.

We recently validated the usefulness of A-F mnemonic in patients with non-ST-segment elevation ACS [23]. Analysis of 916 patients with suspected NSTE-ACS showed a strong correlation between echocardiographic abnormalities in points B-F of A-F mnemonic with other than coronary ethology of chest pain.

In summary we conclude that: 1 . Limited echocardiography with A-F mnemonic is useful in patients with suspected acute coronary syndrome. It allows both confirmation of acute myocardial ischemia and detection of the other life-threatening cardiac conditions requiring definite treatment. 2 . This 5 minutes exam covers a sufficient spectrum of morphological and functional abnormalities of the heart, great vessels and adjacent structures, which can alter the diagnostic and therapeutic management in suspected ACS. 3. Residents with a basic knowledge of echocardiography and short training can perform a reliable initial TTE assessment according to A-F mnemonic

\section{Study limitations and potential pitfalls}

Patients with difficult acoustic window (4.3\%) were excluded from final analysis because of un-interpretable ultrasound images.

Cardiology consultants did not repeat the study, but reviewed the stored digital 10 -seconds video clips, recorded by residents.

Echocardiographic evidence of new regional wall motion abnormalities is one of the diagnostic criteria for an acute myocardial infarction (only when combined with detection of rise and/or fall of cardiac biomarkers) whereas impaired left ventricular global systolic function is a factor related with a poor prognosis. Detection of RWMAs may be challenging especially for non-echocardiographers. Although detection of post-MI scar usually does not cause difficulties (end-diastolic wall thickness $<5 \mathrm{~mm}$, hyperechogenicity), differentiation between preexisting and new hypokinesia may be difficult. Therefore it must be stressed out that echocardiography is only an imaging modality used in the diagnostic process among the others and does not replace clinical reasoning.

\section{Competing interests}

The authors declare that they have no competing interests.

\section{Authors' contribution}

DS: designed the study, supervised data collection, performed the analysis, drafted the manuscript, takes responsibility for the paper as a whole. KN: provided statistical advise on study design and analyzed the data, performed the analysis, took part in manuscript preparation, contributed substantially to the revision of the manuscript. PA: took part in manuscript preparation, contributed substantially to the revision of the manuscript. All authors read and approved the final manuscript.

\section{Acknowledgments}

We would like to express our gratitude to Andrzej Kot, PhD, from University of Science and Technology in Krakow, Faculty of Mechanical Engineering and Robotics, Automatic Control Department, for his help, useful comments and contribution to our study and statistical analyses.

\section{Author details}

'The Department of Interventional Cardiology, John Paul 2nd Hospital, Pradnicka 80, 31202 Cracow, Poland. ${ }^{2} 2$ nd Clinic of Anaesthesiology and Intensive Care, Warsaw Medical University, Warszawa, Poland.

Received: 27 January 2015 Accepted: 17 March 2015

Published online: 26 March 2015

\section{References}

1. Bhuiya FA, Pitts SR, McCaig LF. Emergency department visits for chest pain and abdominal pain: Unites States 1999-2008. NHS Sata Brief. 2010; 43.

2. Thygesen K, Alpert JS, Jaffe AS, Simoons ML, Chaitman BR, White HD, et al. Third universal definition of myocardial infarction. Eur Heart J. 2012;33:2551-67.

3. Steg GP, James SK, Atar D, Badano LP, Blomstrom-Lunquist C, Borger MA, et al. ESC Guidelines for the management of acute myocardial infarction in patients presenting with ST-segment elevation. The Task Force on the management of ST-segment elevation acute myocardial infarction of the European Society of Cardiology. Eur Heart J. 2012;33:2569-619.

4. Hamm CW, Bassand J-P, Agewall S, Bax J, Boersma E, Bueno H, et al. ESC Guidelines for the management of acute coronary syndromes in patients presenting without persistent ST-segment elevation. The Task Force for the management of acute coronary syndromes in patients presenting without persistent ST-segment elevation of European Society of Cardiology. Eur Heart J. 2011;32:2999-3054.

5. Cheitlin MD, Armstrong WF, Aurigemma GP, Beller GA, Bierman FZ, Davis JL, et al. ACC/AHA/ASE 2003 Guideline update for the clinical application of echocardiography: summary article. A report of the American College of Cardiology/American Heart Association Task Force on Practice Guidelines (ACC/AHA/ASE Committee to Update the 1997 Guidelines for the Clinical Application of Echocardiography). Circulation. 2003;108:1146-62.

6. Shah BN, Ahmadvazir S, Pabla JS, Zacharias K, Senior R. The role of urgent transthoracic echocardiography in the evaluation of patients with acute chest pain. Eur J Emerg Med. 2012;19(5):227-83.

7. Labovitz A, Noble VE, Bierig M, Goldstein SA, Jones R, Kort S, et al. Focused cardiac ultrasound in the emergent setting: a consensus statement of the American Society of Echocardiography and American College of Emergency Physicians. JASE. 2010;23(12):1225-30.

8. Sobczyk D, Andruszkiewicz P. Simple mnemonic for focused cardiac ultrasound examination in an emergency. Eur J Anaesthesiol. 2014;31(9):505-6.

9. Arntfield RT, Millington SJ. Point of care cardiac ultrasound applications in the emergency department and intensive care unit - a review. Current Cardiol Rev. 2012;8:98-108.

10. Frenkel O, Riguzzi C, Nagdev A. Identification of high-risk patients with acute coronary syndrome using point-of-care echocardiography in the ED. Am J Emerg Med. 2014. http://dx.doi.org?10.1016/j.ajem.2014.03.003.

11. Ferrada P, Evans D, Wolfe L, Anand RJ, Valquri P, Mayglothing J, et al. Findings of a randomized controlled trial using limited transthoracic echocardiogram (LTTE) as a hemodynamic monitoring tool in the trauma bay. J Trauma Acute Care Surg. 2014;76(1):31-7.

12. Ferrada P, Anand RJ, Whelan J, Aboutanos MA, Duane T, Malhotra A, et al. Limited transthoracic echocardiogram: so easy any trauma attending can do it. J Trauma. 2011;71(5):1327-31.

13. Pershad J, Myers S, Plouman C, Rosson C, Elam K, Wan J, et al. Bedside limited echocardiography by the emergency physician is accurate during evaluation of the critically ill patients. Pediatrics. 2004;114(6):e667-71.

14. Beaulieu Y. Bedside echocardiography in the assessment of the critically ill. Crit Care Med. 2007;35(5 Suppl):S235-49.

15. Vignon P, Mucke F, Bellec F, Marin B, Croce J, Brouqui T, et al. Basic critical care echocardiography: validation of a curriculum dedicated to noncardiologist residents. Crit Care Med. 2011;39(4):636-42. 
16. Mjolstad OC, Andersen GN, Dalen H, Graven T, Skjetne K, Kleinau JO, et al. Feasibility of reliability of point-of-care pocket-size echocardiography performed by medical residents. Eur Heart J Cardiovasc Imaging 2013;14(12):1195-202.

17. Frederiksen CA, Juhl-Olsen P, Andersen NH, Sloth E. Assessment of cardiac pathology by point-of-care ultrasonography performed by a novice examiner is comparable to the gold standard. Scand J Trauma Resusc Emerg Med. 2013; doi:10.1186/1757-7241-21-87.

18. Muniz Pazeli J, Fagundez Vigidal D, Cestari Grossi T, Silva Fernandes NM, Coluqnati F, Baumquartz de Paula R, et al. Can nephrologists use ultrasound to evaluate the inferior vena cava ? A cross-sectional study of the agreement between a nephrologist and a cardiologist. Nephron Extra. 2014;4(1):82-8.

19. Popescu BA, Andrade MJ, Badano LP, Fox KF, Flachskampf FA, Lancelotti P, et al. European Association of Echocardiography recommendations for training, competence, and quality improvement in echocardiography. Eur J Echocardiogr. 2009;10(8):893-905.

20. Panaoulas VF, Daigeler AL, Malaweera AS, Lota AS, Baskaran D, Rahman S, et al. Pocket-size hand-held ultrasound as an adjunct to clinical examination in the hands of medical students and junior doctors. Eur Heart J Cardiovasc Imaging. 2013;14(4):323-39.

21. Spencer KT, Anderson AS, Bhargava A, Bales AC, Sorrentino M, Furlong $K$ et al. Physician-performed point-of-care echocardiography using a laptop platform compared with physical examination in the cardiovascular patient. Am J Coll Cardiol. 2001;37:2013-8.

22. Manasia AR, Nagarj HM, Kodalj RB, Croft LB, Oropello JM, Kohli-Seth R, et al. Feasibility and potential clinical utility of goal-directed transthoracic echocardiography performed by noncardiologist intensivists using a small hand-carried device (SonoHeart) in critically ill patients. J Cardiothorac Vasc Anesth. 2005;19(2):155-9.

23. Sobczyk D, Nycz K, Żmudka K. Usefulness of limited echocardiography with A-F mnemonic in patients with suspected non-ST-segment elevation acute coronary syndrome. Pol Arch Med Wewn. 2014;124(12):688-94.

\section{Submit your next manuscript to BioMed Central and take full advantage of:}

- Convenient online submission

- Thorough peer review

- No space constraints or color figure charges

- Immediate publication on acceptance

- Inclusion in PubMed, CAS, Scopus and Google Scholar

- Research which is freely available for redistribution 